\title{
Novel Functional Association of Serine Palmitoyltransferase Subunit 1-A Peptide in Sphingolipid Metabolism with Cytochrome P4501A1 Transactivation and Proliferative Capacity of the Human Glioma LN18 Brain Tumor Cell Line
}

\author{
T. Yerokun ${ }^{1 *}$, and J. Stewart ${ }^{2}$ \\ ${ }^{1}$ Department of Biological Sciences, Clark Atlanta University, Atlanta, GA 30314, USA \\ ${ }^{2}$ Office of the Dean, School of Computer, Mathematical and Natural Sciences, Morgan, State University, Baltimore, MD 21251, USA \\ *Correspondence to Dr. T. Yerokun. Email: doctortoks@ aol.com
}

Received: 05 January 2005 / Accepted: 06 July 2006 / Published: 30 September 2006

\begin{abstract}
Some chemical modulators of cytochrome P4501A1, Cyp1A1, expression also perturb the activity of serine palmitoyltransferase, SPT, a heterodimeric protein responsible for catalyzing the first reaction in sphingolipid biosynthesis. The effect of altered SPT activity on Cyp1A1 expression has generally been attributed to changes in the composition of bioactive sphingolipids, generated downstream in the SPT metabolic pathway, but the precise mechanism remains poorly defined. A generally accepted model for chemical-induced transactivation of the Cyp1A1 gene involves intracellular signaling mediated by proteins including the arylhydrocarbon receptor, AhR, whose interaction with the 90 kilo Dalton heat shock protein, Hsp90, is essential for maintaining a high affinity ligandbinding receptor conformation. Because ligand-induced Cyp1A1 expression is important in the bioactivation of environmentally relevant compounds to genotoxic derivatives capable of perturbing cellular processes, binding to Hsp90 represents an important regulatory point in the cytotoxicity process. In the present study, based on evidence that indicates subunit 1 of serine palmitoyltransferase, SPT1, interacts with Hsp90, both ligand-induced Cyp1A1 transactivation and capacity for proliferation were evaluated using the wild type Glioma LN18 human brain cancer cell line and its recombinant counterparts expressing green fluorescent SPT1 fusion proteins. Exposure to the prototypical Cyp1A1 inducer, 3-methylcholanthrene, 3-MC, resulted in the translocation of SPT1 from a primarily cytoplasmic domain to sites of focal adhesion complexes. Immunolabel for Hsp90, which was dispersed throughout the cell, became primarily cytoplasmic, while the distribution of AhR remained unaffected. When compared to the wild type, cells transfected with recombinant SPT1-GFP vectors had significantly attenuated levels of 3-MC-induced Cyp1A1 mRNA, as determined by quantitative reverse transcription PCR. Although all the Glioma cell lines exhibited mitogenic proliferative response in dose response assay with the potent Cyp1A1 inducers 3-MC, 2,3,7,8tetrachlorodibenzo-p-dioxin (TCDD) and benzo [k] fluoranthene, BKF, only the recombinant cell line designated 75SPT1-GFP, which was transfected with a mutant deletion of SPT1, retained its proliferative capacity at the highest PAH doses used in this study. The results suggest that overexpressing SPT1 as a green fluorescent fusion protein has a modulating effect on the transactivation of Cyp1A1. This is possibly due to SPT1 interacting with Hsp90 to modulate AhR-Hsp90 interaction, and altering downstream events such as in downregulating the transactivation and metabolic activity of Cyp1A1. This is supported by the fact that the -75SPT1-GFP recombinant cell line, with much lower capacity for Cyp1A1 induction, exhibited sustained mitogenic response to high doses of AhR ligands, but not the Cyp1A1 inducible wild type. Conceivably, the effect mediated by SPT1 on the AhR signaling pathway is an important underlying factor contributing to variability in Cyp1A1 gene expression and consequently, cytotoxic response to environmentally relevant compounds that pose risk to human health.
\end{abstract}




\section{Introduction}

The polycyclic aromatic hydrocarbon (PAH), 3-MC, an environmental pollutant, differentially induces Cyp1A1 in human cell lines $[1,2]$. Members of the Cyp450 family of enzymes, catalyze the bioactivation of a very broad spectrum of compounds to genotoxic derivatives capable of perturbing cellular processes including, signal transduction, cell proliferation or, inducing apoptosis, multidrug resistance phenotype, as well as, leading to neoplastic transformation. A mechanism for transcriptional activation of Cyp1A1 involves an initial binding of ligand to cytoplasmic aryl hydrocarbon receptor (AhR), which, in the unbound state, exists as a complex with the 90 kilo Dalton heat shock protein (Hsp90) and other signaling proteins [3, 4]. When activated, AhR undergoes conformational change, dissociates from Hsp90 and translocates into the nucleus [5-7]. Inside the nucleus, AhR dimerizes with the ARNT to form a complex capable of binding to response elements such as the xenobiotic response element (XRE), in the promoter region of target genes, mediating the transcription of genes involved in xenobiotic metabolism and proliferation [8]. The potent induction of Cyp1A1 by 3-MC is by a low catalytic efficiency, indicative of strong ligand/receptor binding. It is increasingly evident that, sphingolipids (SLs), in addition to providing essential structural integrity to cells, play important roles as key mediators of cellular response to xenobiotics. In the de novo SL biosynthesis pathway, SPT catalyzes the rate-limiting reaction, condensing L-serine with palmitoyl coenzyme A [9-11]. Subsequent downstream reactions then generate long-chain SLs including sphinganine (Sa), sphingosine (So) and dihydroceramide, as well as ceramide (Cer), the precursor of such complex SLs as glucosylceramide (GlcCer) and sphingomyelin (SM). These SLs are bioactive, implicated as second messenger molecules in diverse biological processes [12], linked to the regulation of metabolic enzymes, while, on the other hand, overexpression of some metabolic enzymes has been found to modulate levels of these bioactive SL species [13-15]. More recently, certain chemicals that alter the expression of SPT have been found to also alter Cyp1A1 expression. Exposure of human hepatocarcinoma, HepG2, to Cer generated via the SPT metabolic pathway, or inhibition of SPT with myriocin, have been reported to modulate 3-MC-induced Cyp1A1 expression [14]. Other agents that modulate SPT activity, including resveratrol, 4-HPR and hypoxia, also modulate Cyp1A1 expression [16-19]. How these agents exert overlapping effects on pleiotropic metabolic pathways, in this case SL and xenobiotics, whether mediated primarily through perturbation of cellular SL composition, modulation of signal transduction pathways that regulate cellular response processes, or a combination of factors, remain to be fully established.

Toxicological response to PAHs results from the actions of a complex network of metabolic pathways and crosstalk among diverse signaling pathways. Such interactions provide considerable opportunity for greater specificity in regulating gene expression in toxicological response events. Chaperone proteins such as Hsp90, with a variety of protein-binding modules that facilitate proteinprotein interactions for forming signaling heterocomplexes play important role in integrating the multiple signaling pathways, which regulate specific and appropriate cellular response to diverse stimuli. Such interaction with Hsp90 is thought to contribute to the dynamic ability for crosstalk between $\mathrm{AhR}$ and other signaling proteins in diverse cellular processes [20]. Agents that modulate heterocomplex formation with Hsp90 may alter signaling mediated via AhR with potential consequences on gene transactivation and other cellular processes. For example, in the absence of exogenous agonists, protracted AhR activity, from excessive or prolonged mitogenic signaling, is thought to be capable of stalling $\mathrm{G}_{1}$ phase progression. This effect is similar to that mediated in cultured rat hepatoma cells by the prototypical AhR ligand and activator 2,3,7,8-tetrachlorodibenzo-p-dioxin (TCDD) [21]. In contrast, a negative feedback mechanism that leads to suppression of a prolonged AhR activity thought to be involved in the depletion of endogenous AhR agonist by Cyp1A1 under normal physiological conditions [21]. While much is known about the biochemistry of SLs and PAHs, understanding the possible modulating effect the interaction of the metabolic pathways has on cellular response may help provide more insight into the molecular mechanism of action of xenobiotics. The convergence of modern molecular biology approaches with the physiological context provided by decades of biochemical studies of the xenobiotic and SL metabolic pathways, broaden the opportunity for better understanding underlying mechanisms by which the interaction of the metabolic pathways may contribute to regulate complex cellular responses, particularly response to toxic agents that may cause human neoplasia.

\section{Materials and Methods}

\section{Chemicals}

3-MC, TCDD (dioxin), benzo [k] fluoranthene (BKF) and the dietary carcinogen 2-amino-1-methyl-6phenylimidazo[4,5-b]pyridine (PhIP) were purchased from Sigma Chemical Company (St. Louis, MO).

\section{Cell Culture and Treatment}

The wild-type human brain tumor glioblastoma LN18 cell line, obtained from Dr. Erwin G. van Meir (Winship Cancer Institute, Emory University, Atlanta, GA), was cultured in Dulbecco's minimum essential medium (DMEM) supplemented with $10 \%$ fetal bovine serum (FBS) and $100 \mathrm{U} / \mathrm{ml}$ penicillin and $100 \mathrm{ug} / \mathrm{ml}$ streptomycin, and grown at $37^{\circ} \mathrm{C}$ in a humidified atmosphere of $5 \% \mathrm{CO}_{2}$. The cells were routinely cultured in $100 \mathrm{~mm}$ dishes, or plated overnight at $3 \times 10^{4}$ cells per well of a 4-well chamber slide (Nalge Nunc, VWR, West Chester, PA), when stained for confocal imaging. For treatments, cells 
were passaged by exposure to trypsin/EDTA and plated in DMEM without FCS at densities of $3 \times 10^{5}$, or $5 \mathrm{X}$ $10^{4}$ cells per well of a 6-well or 96-well plate, respectively. Prior to treatment, cells were allowed to adhere and acclimatize in the serum-free medium for $3 \mathrm{~h}$, rinsed in PBS before being treated. Stock solutions of all test chemicals were prepared in dimethyl sulfoxide (DMSO) then dissolved in serum-free DMEM, to the final concentrations used for cell treatment. Control treatment was with $<0.2 \%$ DMSO in DMEM. Cell treatment was followed by a phosphate buffered saline (PBS) wash, before harvesting for cell and molecular analysis protocols.

\section{Antibodies and Staining Reagents}

Phalloidin dye for detecting cytoskeletal F-actin fibers, nuclei Hoescht 33343 dye, goat anti-mouse and goat anti-rabbit secondary antibodies conjugated with Alexa Fluor fluorophore, were obtained from Molecular Probes, Inc. (Eugene, OR). Primary antibody against paxillin was purchased from BD Transduction Laboratories (Palo Alto, CA), and the anti-Hsp90 antibody was purchased from Stressgen Biotechnologies (Victoria, BC Canada). The rabbit anti-human anti-SPT1 antibody was obtained from Dr. David J. Uhlinger and has been characterized in detail in a previous publication [22].

\section{Staining for Immunofluorescence Confocal Microscopy}

Cells cultured overnight on chamber glass slide were fixed with $3 \%$ formaldehyde in 1x PBS, pH 7.4, at room temperature for 10 minutes. Fixed cells were rinsed in PBS supplemented with 10\% FBS (serum-PBS) and used in the staining procedure. This involved incubating fixed cells for $1 \mathrm{~h}$ with primary antibody diluted 1000 -fold in serum-PBS, rinsing in serum-PBS for $10 \mathrm{~min}$ then resuspending fixed cells in fluorophore-conjugated secondary antibody, again diluted 1000 -fold in serum PBS for $1 \mathrm{~h}$, all at room temperature. Stained cells were rinsed twice in serum-PBS, followed by a final rinse in PBS and mounting in Fluoromount G (Southern Biotechnology Associates, Inc., Birmingham, $\mathrm{Al}$ ), to preserve fluorescent emission. Recombinant GFP cells were fixed and mounted directly.

\section{Immunofluorescence Confocal Imaging of SPT in Cultured Cells}

Labeled cells mounted on slides were observed with a Zeiss LSM510 inverted microscope with a Zeiss PlanApochromat $43 \times$ oil immersion objective lens. The microscope was equipped with argon, helium and neutron (HeNe) lasers which allow simultaneous 3channel fluorescence imaging and visualizing single or multiple band excitation and emission of wavelengths in the green, red and blue regions. Images collected with the resident Zeiss confocal microscope software were processed using Adobe Photoshop.
Primer Design, Vectors, RNA Preparation and Quantitative Real Time Reverse Transcriptase PCR (Q-RTPCR)

The primer pairs used for specifically amplifying the SPT1 and Cyp1A1 genes were based on published cDNA sequence data:

CYP1A1 forward primer: 5'TGGTCTCCCTTCTCTACACTCTTGT3', and

CYP1A1 reverse primer:

5'ATTTTCCCTATTACATTAAATCAATGGTTCT3', designed to amplify a 141 base pair fragment from cypla1 [23]. SPT1 forward primer: 5'AGGAGTCACTGAACACTATG3'

SPT1 reverse primer: 5'AGCTCTCTCCAGTTCTTCCT3', designed to amplify the full-length 735 base pair fragment of SPT1 [24]. The pEGFP-N1 and $\mathrm{pCMV} / \mathrm{myc} / \mathrm{mito}$-plasmid vectors from the pShooter-system were from Invitrogen (Carlsbad, CA). The pEGFP vector has a multiple cloning site upstream of the GFP sequence, while the $\mathrm{pCMV} / \mathrm{myc} /$ mito vector has a targeting sequence derived from subunit VIII of human cytochrome c oxidase, a 72 amino acids and 8129 Dalton peptide fragment that allows efficient targeting of the GFP to the mitochondria. Total cellular RNA was isolated from cultured cells with TRizol (Invitrogen, San Diego, CA). Real time PCR with fluorescence detection system was used to determine gene expression levels. Briefly, total RNA was processed by incubation with Dnase, to remove any contaminating DNA. Using reagents from the Superscript III Platnum SYBR Green One Step QRT-PCR kit (Invitrogen, San Diego, CA), and the primer set described previously, processed RNA samples were subjected to reverse transcription and amplification, following published protocol [23]. Amplification of the $18 \mathrm{~S}$ rRNA was used as reference control to monitor total mRNA copy numbers amplified per RNA sample.

\section{Functional Engineering and Transfection of SPT1-GFP Vectors}

The GFP gene and its enhanced derivatives encoding naturally fluorescent light emitting chromophores have become a popular living marker for transiently and stably transfected clones in many studies [25]. However, because transfection of the empty GFP vector has the tendency to be toxic or potentiate cytotoxic response, the mitochondriaGFP (mito-GFP) vector was used as control for transfected cells. The mito-GFP fusion protein is expressed in recombinant cells as a mitochondria membrane-associated protein that is biologically non-functional in the target cell. Recombinant vectors carrying full length and mutant deletions of the human SPT1 sequence generated by reverse transcription-PCR, and expressed as green fluorescent fusion proteins (GFP) were constructed with the pEGFP-N1 mammalian expression vector (Invitrogen, San Diego, CA). The SPT1 cDNA was ligated into the vector at a site upstream of the GFP sequence. One day prior to transfection, cells were split and plated overnight at $5 \times 10^{4}$ cells per well of a 6 -well culture plate. The 
following day, cells were rinsed with PBS and resuspended in serum-free DMEM. These cells were then transfected with recombinant GFP vector DNA, using $1 \mathrm{ul}$ lipofectamine transfection reagent (Clontech Laboratories, Inc., Palo Alto, CA), according to manufacturer's directions.

Immunoprecipitation and SDS-polyacrylamide Gel Electrophoresis (SDS-PAGE)

This was used to assess whether SPT1 exists in heterocomplex with the stress-responsive paxillin and Hsp90 proteins in lysate of stressed cells. Briefly, heatshocked cells in culture plates were rinsed with PBS, scraped in $5 \mathrm{ml}$ PBS with a rubber policeman and pelleted in $15 \mathrm{ml}$ tubes, which were centrifuged at 2000 rpm for $3 \mathrm{~min}$. While on ice, the cell pellet was suspended in $1 \mathrm{ml}$ lysis buffer $(0.25 \mathrm{M}$ Sucrose, $5 \mathrm{mM}$ $\mathrm{CaCl}_{2}, 25 \mathrm{mM}$ Hepes, pH 8.0, $10 \mathrm{mM}$ Tris- $\mathrm{HCl}, \mathrm{pH}$ 7.5, $5 \mathrm{mM}$ EDTA, $1 \mathrm{mM}$ PMSF, $20 \mu \mathrm{g} / \mathrm{ml}$ Leupeptin; 10 $\mu \mathrm{g} / \mathrm{ml}$ Aprotinin; $10 \mu \mathrm{g} / \mathrm{ml}$ Pepstatin). The cells were fractionated by 10 passages through a 25 -gauge syringe. The homogenate was centrifuged at $1000 \mathrm{x}$ g for $10 \mathrm{~min}$ and the supernatant used as total cell lysate. All fractions collected were aliquoted for determining protein concentrations and the rest stored at $-80^{\circ} \mathrm{C}$. A $1 \mathrm{~g}$ equivalent of cell lysate protein was used for immunoprecipitation, by being mixed separately, with each of $5 \mu \mathrm{g}$ of anti-SPT1, anti-Hsp90, or anti-paxillin antibody. This was mixed with $60 \mu \mathrm{l}$ of a $50 \%$ PBS slurry of protein A-sepharose (Amersham, Piscataway, NJ), at $4^{\circ} \mathrm{C}$, overnight. The beads were washed by centrifugation three times with $1 \mathrm{ml}$ ice-cold PBS containing protease inhibitor. Proteins bound to the beads were eluted with 80 $\mu \mathrm{l}$ sample loading buffer at $65^{\circ} \mathrm{C}$ for $10 \mathrm{~min}$. Separation of sample proteins either from total cell lysate (about $50 \mu \mathrm{g}$ protein) or immunoprecipitated fractions was accomplished by mixing each sample with an equal volume of gel-loading buffer and boiling at $90^{\circ} \mathrm{C}$ for 5 min. The denatured protein sample was loaded on a $12 \%$ SDS-PAGE and run at 100 volts until the dye front reached the bottom of the gel.

\section{Western Blotting}

PAGE samples were transferred from gel to nitrocellulose filter using Tris-glycine buffer prepared in $20 \%$ methanol as the transfer medium, for $1 \mathrm{~h}$ at 100 volts (constant), in a transfer unit (BIO RAD, Hercules, CA). The blot was blocked overnight at $4^{\circ} \mathrm{C}$, with a $5 \%$ milk solution in tris-buffered saline supplemented with $0.1 \%$ Tween-20 (TBST). The filter was then incubated with primary antibody diluted 1000 -fold in $1 \%$ milk solution in TBST, overnight at $4^{\circ} \mathrm{C}$. Filters were rinsed twice in TBST for 20 minutes then blocked in 5\% milkTBST for $1 \mathrm{~h}$ at room temperature, followed by incubation at room temperature for $1 \mathrm{~h}$, in $1 \%$ milk/TBST solution containing peroxidase-conjugated secondary antibody at 1000-fold dilution. Finally, filters were rinsed in TBST and the conjugated peroxidase developed by incubating filters in Supersignal West Pico chemiluminiscent reagent (Pierce, Rockford, IL). The peroxidase activity on filters was visualized with a Fluor-S multi-imager (BioRad, Hercules, CA).

\section{Cellular Viability/Proliferation Assay}

This was performed using a modification of the microculture tetrazolium assay, with the reagent WST-1 (Roche Diagnostics, Indianapolis, USA). This reagent, similar to MTT, contains a tetrazolium salt, which is cleaved by metabolic enzymes to a water-soluble formazan. The cleavage is accompanied by change in color from light red to dark red, which directly correlates with the number of metabolically active cells in culture. This allowed for the quantitative colorimetric assessment of cellular viability and proliferation following treatment. Cells were seeded at $5 \times 10^{4}$ per well of a 96-well culture plate, in serum-free DMEM, and allowed to adhere over a 12-h period, in a cell culture incubator at $37^{\circ} \mathrm{C}$ and $5 \%$ $\mathrm{CO}_{2}$. The DMEM medium was removed and cells reincubated in DMEM with test chemical. Treatment was terminated, by rinsing cells in PBS and re-incubating in DMEM supplemented with WST-1. After $1 \mathrm{~h}$ incubation with WST-1, absorbance in each well was measured with a microplate reader at $480 \mathrm{~nm}$. The absorbance of the formazan product at a wavelength of $480 \mathrm{~nm}$, after correcting for background from control wells, was used as an index of cell proliferation. Percentage of viable cells was computed as follows:

$$
\% \text { viable cell }=\frac{\text { Absorbance } 480 \mathrm{~nm} \text { (sample) }}{\text { Absorbance } 480 \mathrm{~nm} \text { (Untreated cells) }}
$$

\section{Tandem Mass Spectometry}

Determination of cellular SL composition followed the detailed procedure published in the study of Sullards et al [26]. The data generated were normalized per mg protein in cell extracts examined. Each sample was run in triplicates and the mean and standard deviation computed for each sample.

\section{Results}

Endogenous SPT1, Hsp90, SPT1- and AhR-GFP fusion proteins localize to cytoplasmic domain, and 3-MC induces translocation of SPT1 to Focal Adhesion Complex in Glioma LN18 cells: Using epitope-specific antibodies in fluorescence immunolabeling, images from confocal microscopy in Figure 1 indicates that endogenous SPT1, Hsp90, SPT1- and AhR-GFP fusion proteins all localize to cytoplasmic cellular domain, while paxillin localizes, primarily, to FA complexes (Fig. 1, left column, rows 1-4). The distribution of endogenous proteins is consistent with the cellular localization expected for these proteins. 
Untreated

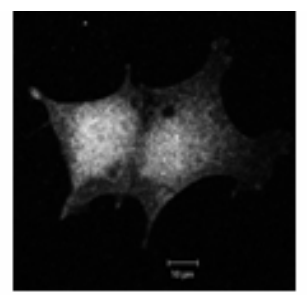

SPT1

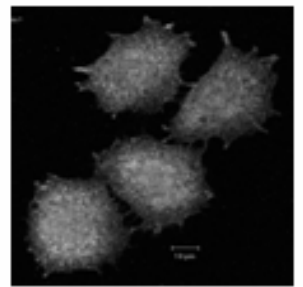

Hsp90

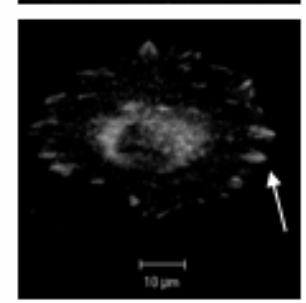

Paxillin
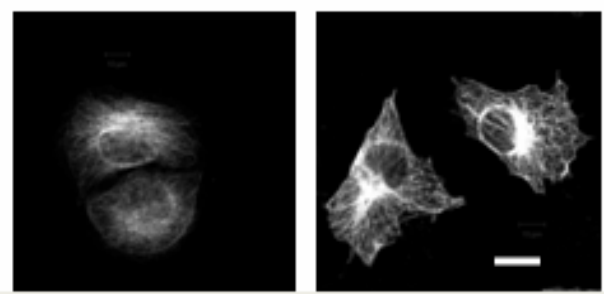
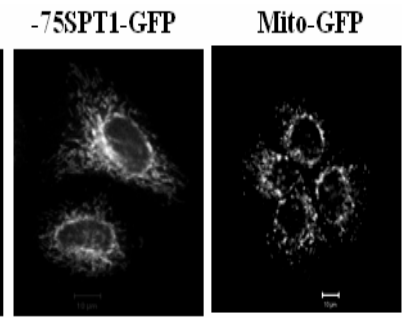

AlR-GFP
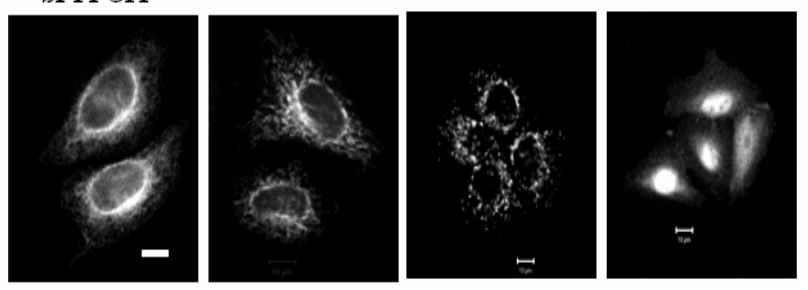

Figure 1: Sub-cellular and co-locilization of SPT1, Hsp90, paxillin, microtubule and GFP-fusion proteins of SPT1 and AhR by immunofluorescence confocal microscopy: The cytoplasmic localization of endogenous SPT1 was determined in immunofluorescence staining of fixed cells (Fig. 1, row 1, left column), and shows the characteristic distribution associated with microsomal fraction of the cell. The Hsp90 had dispersed cytoplasmic distribution, paxillin had cytolasmic and characteristic localization to sites of focal adhesion complexes (arrow), while microtubule staining displayed distinct tubular network (Fig. 1, rows 2, 3 and 4). In cells treated with 3-MC for $3 \mathrm{~h}$, both SPT1 and Hsp90 displayed differential staining patterns when compared to endogenous distribution, while the staining patterns for paxillin and microtubule remained essentially the same. The 3-MC resulted in translocation of SPT1 to sites of FA (Fig. 1, row 1, right column), there were no Hsp90 immunolabel in the nuclei region, while the perinuclear and FA sites became more intensely labeled (Fig. 1, row 2, right column. The distribution of green fluorescent proteins expressed in Glioma LN18 cells transfected with SPT1-GFP, -75SPT1-GFP, mitoGFP and AhR-GFP recombinant vectors was viewed in cells grown on culture slides, fixed and mounted, using confocal microscope. The SPT1-GFP, -75SPT1-GFP and mito-GFP recombinant cells displayed the cytoplasmic fluorescence of the fusion proteins (Fig. 1, row 5), while the AhR-GFP recombinant cells displayed both nuclei and cytoplasmic fluorescence of the fusion protein. The cellular distribution seen with the fusion proteins is consistent with the cytoplasmic localization of wild type proteins. Horizontal bars $=10 \mu \mathrm{m}$

Stably transfected clones of SPT1-GFP recombinant cells selected with G418 and clonally expanded were used in the current study. Similar to the endogenous SPT1, there is cytoplasmic distribution of both the full length SPT1 green fluorescence fusion protein (Fig. 1, row 5, SPT1GFP) and the -75SPT1-GFP deletion mutant (Fig. 1, row 5). However, the nuclei distribution of endogenous SPT1 is absent with the recombinant SPT1-GFP fusion proteins (Fig. 1, row 5, columns 1 and 2). Endogenous Hsp90 distribution in untreated cells (Fig. 1, row 4), shows dispersed cellular distribution over cytoplasmic and nuclei regions. In cells treated for $24 \mathrm{~h}$ with $5 \mu \mathrm{M}$ 3-MC, endogenous SPT1 becomes translocated to FA sites (Fig. 1, right column, row 1, arrow), Hsp90 becomes primarily localized to cytoplasm with some in A sites (Fig. 1, right column, row 2, arrow), while paxillin and microtubules retained their localization to FA sites and cytoplasm, respectively (Fig. 1, right column, rows 3 and 4).

SPT1 is co-precipitated with Hsp90 from lysate of stressed cells and identified in lysate of recombinant cells: Because of the apparent capacity of SPT1 to respond to stress as evidenced by its translocation to FA sites, its ability to interact with the known stress responsive molecular chaperone Hsp90 was assessed by combined immunoprecipitation/Western blot analysis. Antibodies against SPT1and Hsp90 were used for immunoprecipitating the respective proteins, present either alone or as heterocomplexes in cell lysates. Following immunoprecipitation and PAGE fractionation, Western blots screened with anti-SPT1 antibody identified protein bands with an apparent size of about $50 \mathrm{kDa}$, corresponding to SPT1, in fractions from total cell lysates of a positive control and heat shocked wild type Glioma, respectively (Fig. 2, P and G). Cell lysate fraction screened with pre-immune antibody, as negative control, generated no signals (Fig. 2, N). Protein bands of apparent size 50 $\mathrm{kDa}$, corresponding to SPT1, were also detected in the immunoprecipitate fractions generated using anti-SPT1, paxillin and -Hsp90 antibodies, respectively (Fig. 2, S, H and X). When screened by Western blot analysis for SPT1, lysates from SPT1-GFP and -75SPT1-GFP recombinant cells all indicated the presence of endogenous SPT1 protein bands of an apparent size in the $50 \mathrm{kDa}$ range, corresponding to SPT1 (Fig. 2, F and M). In the same 
lanes, the presence of SPT1-GFP fusion proteins is indicated by a protein band shift of apparent size in the $80 \mathrm{kDa}$ size range (Fig. 2, F and M). This band shift is in agreement with what is expected from the fusion of a 55 $\mathrm{kDa}$ SPT1 and $30 \mathrm{kDa}$ GFP protein fragments.

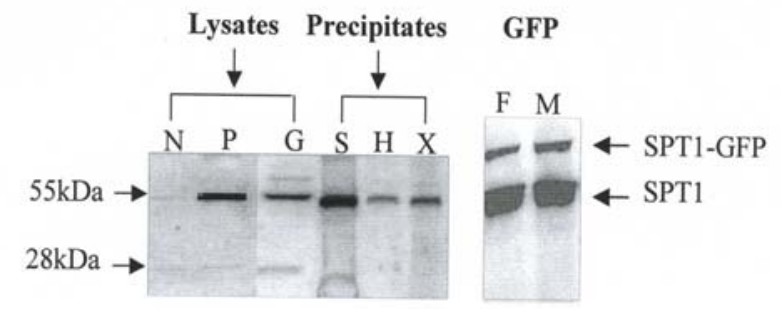

Key: N- negative control; P-positive control; G- Glioma IP: S- anti-SPT1, H- anti-Hsp90, X- anti-Paxillin GFP: F- full length SPT1-GFP; M- -75SPT1-GFP

Figure 2: SPT1 co-precipitation with Hsp90 from total lysate of stressed cells, and band shift as GFP fusion protein: Screening for SPT1 using anti-SPT1 antibody indicates positive bands of apparent size $55 \mathrm{kDa}$, in total cell lysate of a positive control cell line and the Glioma LN18 cell line (Fig. 2, P and G), consistent with the expected protein size for SPT1. In cells subjected to heat shock, cell lysates subjected to immunoprecipitates with anti-SPT1, Hsp90 or paxillin, and then immunoprecipitates screened for SPT1 (Fig. 2, S, H and $\mathrm{X})$, the presence of SPT1 band with apparent size 55 $\mathrm{kDa}$, as in total cell lysate was detected. The presence of endogenous SPT1 or the green fluorescent fusion protein was determined by Western blot analysis of total lysate from transfected cells. When probed with anti-SPT1, there is evidence for the presence of endogenous SPT1 in cell lysates of SPT1-GFP recombinant cells corresponding to the 55 kDalton protein marker, while a band shift for the SPT1-fusion protein indicates a protein of apparent size $80 \mathrm{kDa}$, which is in agreement with the expected size for a fusion protein consisting of $55 \mathrm{kDa}$ SPT1 and $30 \mathrm{kDa}$ GFP.

Wild type and SPT1 recombinant Glioma cells have similar composition of the long chain sphingoid bases sphinganine ( $\mathrm{Sa}$ ), sphingosine (So) and ceramide (Cer): Neuroblastoma cells responding to diverse stress stimuli have been reported to have differences in SL composition, compared to untreated cells [27], hence, residual or induced SL composition may contribute in cellular disposition to the cytotoxic effect of stress stimuli. The SL composition of both wild type and stably transfected recombinant Glioma cells used in this study was determined by mass spectrometry using a previously published approach [26]. As shown in Figure 3, the wild type, stably transfected SPT1-GFP and -75SPT1-GFP, as well as the Mitochondria-GFP recombinant Glioma cells (mito-GFP), all have similar composition of the $\mathrm{Sa}$, So and Cer sphingoid bases, as well as the phosphate derivates for $\mathrm{Sa}$ and So, S-1-P, SaP.

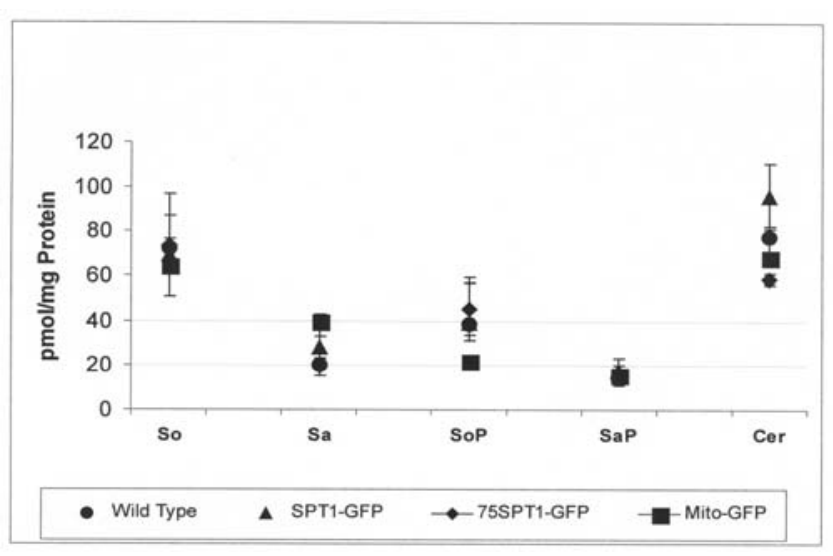

Figure 3: Metabolomic profile of sphingoid base composition in wild type and SPT1-GFP recombinant cells: The SL composition for So, Sa, and Cer shows similar levels in both the wild type and SPT1 recombinant cell lines, as determined by mass spectrometry. The levels of phosphorylated derivatives of $\mathrm{Sa}$ and So were also similar in all the cell lines. The SL composition extracted from untreated lyzed cells was determined by mass spectrometry [27], each sample assayed in triplicates and values reported as picomoles SL contents per $\mathrm{mg}$ of cell protein. The standard deviation for each data set was computed and significant difference by Student t test.

The CyplA1 gene is differentially induced in wild type and SPT1-GFP recombinant cells following exposure to 3$M C$ : The Glioma cell line used in this study derives from human brain known to contain a Cyp4501A1-dependent metabolic system [28], while 3-MC is known to potently induce transactivation of Cyp1A1 via AhR signal pathway [1]. The effect SPT1 expression has on this process in SPT1 recombinant cells was examined by quantitative RTPCR analysis of relative Cyp1A1 mRNA levels in wild type compared to recombinant cells. Figure 4 shows Cyp1A1 induction is significantly attenuated in cells transfected with the full length SPT1-GFP or the -75SPT1GFP deletion mutant vector, but significantly increased in the parental cell line and mito-GFP control cell line, following 3-MC treatment for $24 \mathrm{~h}$. Comparing untreated cells to 3-MC-treated cells, the fold increase in Cyp1A1 copy numbers indicated an increase of about 250 -fold with the wild type cell line, about 100-fold increase for SPT1GFP, about 10-fold increase for -75SPT1-GFP and about 30-fold increase for the mito-GFP. However, when compared to the untreated wild type, the fold increase in Cyp1A1 copy number induced by 3-MC in wild type, SPT1-GFP, -75SPT1-GFP and mito-GFP cells was about 250, 70, 80 and 1000, respectively. This indicates a Cyp1A1 inducibility index at least 3 times higher in either wild type or mito-GFP control, compared to the SPT1-GFP or -75SPT1-GFP recombinant cells. The decreased induction of Cyp1A1 in the SPT1-GFP and -75SPT1-GFP recombinant cell lines suggests that the overexpression of SPT1 as a GFP fusion protein in these cell lines may be interfering with the AhR signaling pathway, probably because the SPT1 protein interacts with Hsp90 to perturb 
AhR/Hsp90 interaction required for a high affinity ligand-binding conformation of AhR.

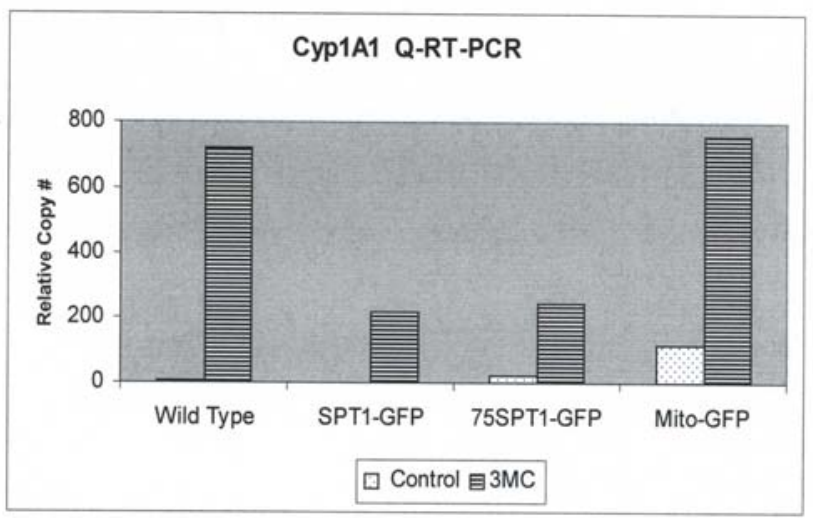

Figure 4: Inducibility of Cyp IAI by 3-MC is attenuated in cells Expressing SPT1 as GFP fusion proteins: Using untreated cells as control and cells exposed to 3-MC over a $24 \mathrm{~h}$ period, cells were harvested, lyzed, total RNA was extracted and used in quantitative RT-PCR. The copy number of Cyp1A1 detected in PCR samples was computed relative to the S18 rRNA gene copy number. When compared to the untreated wild type, the fold increase in Cyp1A1 copy number induced by 3-MC in wild type, SPT1-GFP, -75SPT1-GFP and mito-GFP cells was about 250, 70, 80 and 1000, respectively, showing decreased induction of Cyp1A1 in recombinant cells transfected with SPT1-GFP vectors, but not the mito-GFP.
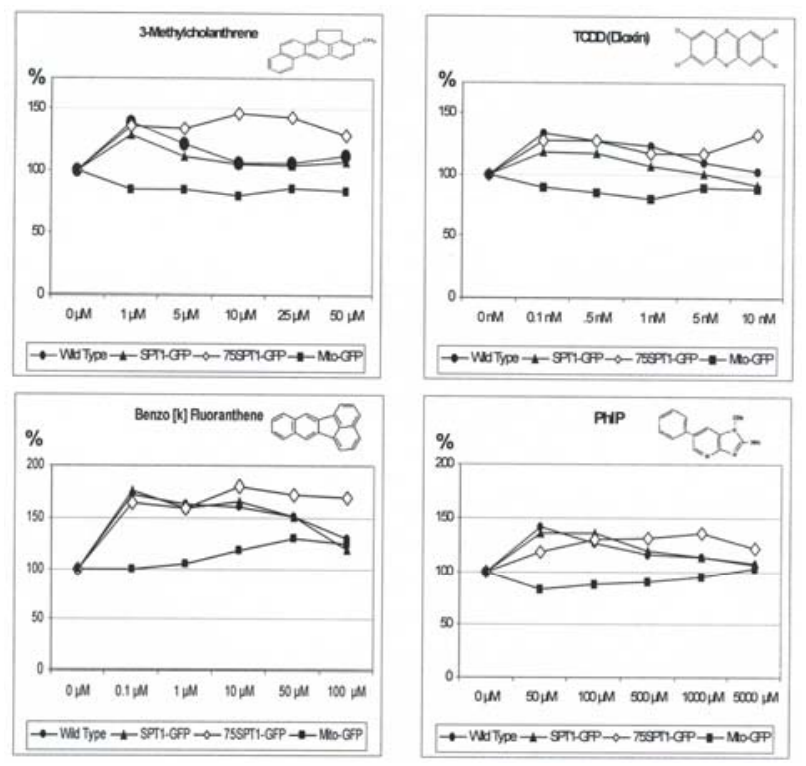

Figure 5: The wild type and recombinant SPTI Glioma cells exhibit differential and dose-dependent proliferative response to $3-M C, T C D D$ and $B K F$, but not PhIP: The polycyclic aromatic hydrocarbons 3-MC, TCDD, BKF and the heterocyclic amine PhIP, are AhR agonists, but the PAHs are more potent Cyp1A1 inducers compared to PhIP. Cellular response to 3-MC $(1-5 \mu \mathrm{M})$, TCDD (0.1-0.5 nM), BKF (0.1-10 $\mu \mathrm{M})$, or PhIP (50-100 $\mu \mathrm{M})$, is clearly a mitogenic response for the wild type,
SPT1-GFP and -75SPT1-GFP cell lines. In contrast, exposure to 3-MC $(50 \mu \mathrm{M})$, TCDD $(10 \mathrm{nM})$ or BKF (100 $\mu \mathrm{M}$ ), is cytotoxic to the wild type, SPT1-GFP and mitoGFP, but not the -75SPT1-GFP cell line. Similar to the MTT assay, cell viability was assessed with WST-1 reagent. Using cell at a density of $5 \times 10^{4}$ per well of a 96-well plate and 6 wells per treatment point, data represents relative percentage of surviving cells as previously described.

Dose response assay shows wild type and SPT1 recombinant Glioma cells exhibit differential proliferative capacities in a dose-dependent manner to the potent Cyp1A1 inducers 3-MC, TCDD and BKF, but not PhIP: The capacity of polycyclic aromatic hydrocarbons and heterocyclic amines including 3-MC, TCDD, BKF and $\mathrm{PhIP}$ to induce Cyp1A1 expression exerting cytotoxic effects has been reported $[1,2]$. When assessed by WST-1 assay, all cell lines showed similar dose-dependent metabolic index of proliferation, following $24 \mathrm{~h}$ exposure to either 3-MC, TCDD or BKF (Fig. 5). In contrast, only the -75SPT1-GFP recombinant cell line maintained a mitogenic proliferative response to the highest doses applied with each of 3-MC $(50 \mu \mathrm{M})$, TCDD $(10 \mathrm{nM})$ or BKF $(100 \mu \mathrm{M})$. The role of SPT1 in determining Cyp1A1 metabolic status of the cell lines and possible contribution to cytotoxic response to high PAH doses is further supported by the fact that exposure to PhIP, a less potent Cyp1A1 inducing heterocyclic amine, whose metabolic activation is known to depend on Cyp1A2 [29], elicited similar proliferative responses, as assessed by WST-1 assay, in all the cell lines (Fig. 5).

\section{Discussion}

Cellular response to stress including response to toxicologically relevant PAHs involves a complex network of metabolic pathways and crosstalk among diverse signaling pathways. Chaperone and adaptor proteins such as Hsp90, with a variety of protein-binding modules that facilitate protein-protein interactions for signaling heterocomplexes play important role in integrating multiple signaling pathways, which regulate specific and appropriate cellular response to diverse stimuli. The interaction of SPT1 with Hsp90 has the potential to modify protein species in signal transducing heterocomplexes, thereby playing a novel role in determining biological outcome from exposure to xenobiotics. The molecular chaperone, Hsp90, is known to exist in heterocomplexes with its co-chaperones to regulate protein folding and trafficking [30] and association with its co-chaperone, p23, is essential to the formation of the AhR heterocomplex. In the absence of ligand, the binding of AhR to Hsp90, with other proteins in the heterocomplex, is required for the cytoplasmic retention of AhR, its protection from proteasomal degradation, and formation of the highaffinity ligand-binding conformation of the receptor [31, 32]. This is thought to enhance AhR signaling and ultimately, contributes to regulate the transactivation of the AhR battery of genes including Cyp1A1. Hence, agents 
capable of disrupting interaction and/or heterocomplex formation with Hsp90, such as SPT1, can perturb the AhR signaling pathway and cause transactivation of Ah battery of genes to be downregulated. Given the critical role Hsp90 plays in ligand binding activity and activation of the AhR, perturbing Hsp90 interaction, such as with SPT1/Hsp90 binding, may modulate the formation of AhR/Hsp90 complexes and mediate an attenuating effect on downstream transactivation events for Cyp1A1 expression, as seen in this study. The AhR-Hsp90 signaling pathway is also implicated in the proliferation of normal cells and the aberrant proliferation of cancer cells. This Hsp90 function is thought to be achieved through the capacity to bind an array of cochaperones including Hop and p23, forming heterocomplex that interacts with client oncogenic proteins such as Her-2, Raf-1, and Akt involved in signal transduction, cell cycle regulation, and apoptosis. Binding to Hsp90 leads to ubiquitination and subsequent proteasomal degradation of these client proteins. Based on the knowledge that SPT1 binds Hsp90, the use of SPT1-GFP recombinant cells in the present study permitted the assessment of the biological and biochemical effects SPT1 expression may have on specific Cyp1A1 gene expression and cellular response to environmental genotoxins. When cells are exposed to chemical agents known to induce the transactivation of Cyp1A1 via AhR signal pathway, it is evident from results of the present study that the expression of SPT1 as a GFP fusion protein attenuates the transactivation of the Cyp1A1 gene. The potent induction of Cyp1 1 1 by $3 \mathrm{MC}$ is considered to be by a lower catalytic efficiency, indicative of strong ligand/receptor binding, while $\mathrm{PhIP}$, a less potent inducer of Cyp1A1 but potent inducer of Cyp1A2, is thought to have a very high catalytic efficiency $(\mathrm{Vmax} / \mathrm{Km})$ for Cyp1A1 induction, indicative of low ligand/receptor binding [29]. When considered with respect to the pattern of Cyp1A1 inducibility in the wild type and SPT1 recombinant cells used in the present study, it appears that the Cyp1A1 metabolic status of the cell lines contributes to cytotoxic response to high PAH doses, probably because perturbation of the AhR signaling pathway in the -75SPT1-GFP cells mediates attenuated Cyp1A1 expression, consequently diminishing the Cyp450 metabolic activity and results in refractive cytotoxic response. Such response will not be expected for chemicals that do not depend on Cyp1A1 for their metabolic activation, as is the case with PhIP, which, at all treatment doses used, elicited similar cytotoxic response in both wild type and SPT1 recombinant cell lines.

The SL metabolic pathway via SPT and generation of bioactive SLs, is also linked to Cyp450 expression and cytotoxic response to chemical agents. Decreased Cer levels have been reported in both human glial cancer cells, as well as, in cancers of other tissue origins, suggesting a role in malignant progression [33, 34]. Although changes in SL metabolism accompanied with altered cellular SL distribution have been reported to influence the expression of members of the Cyp450 family of enzymes, it is unlikely that this is the case for the Glioma cells used in the present study as they all exhibited similar composition for two of the sphingoid bases So and Cer, frequently associated with the effect SLs have on Cyp450 expression. However, it cannot be ruled out that these undergo changes during chemical treatment and that the changes contribute to the gene expression activity or cytotoxic response process.

The human brain is known to contain a Cyp4501A1dependent metabolic system [28], with a potentially important role in the metabolism of endogenous and exogenous compounds, as well as, possibly influencing cellular responses to environmental toxins. The Glioma LN18 cell line used in the current study derives from a common form of brain tumor often resistant to therapeutic intervention [35] and whose sphingolipid (SL) profile has been previously characterized [26]. It was not unexpected for the wild type cell line to be refractive to the cytotoxic effects of the chemical agents used in this study. However, because of the differences in Cyp1A1 levels induced in wild type and SPT1-GFP recombinant cells at sublethal dose of 3-MC, while cytotoxic response to the AhR ligands was only evident at the highest ligand concentrations used, the differential Cyp1A1 expression profile can be more likely implicated as a determining factor in cytotoxic response. The refractive cytotoxic response of -75SPT1GFP recombinant cells to high PAH doses probably reflects the modulatory influence the mutant SPT1 fusion protein is having on signaling via $A h R$, rather than as a consequence of SL composition or changes in SL composition in these cells. When considered within the biochemical context available for the xenobiotic and SL metabolic pathways, the observations made in the present study strongly suggest that the SPT1 protein subunit may have additional functional role in Cyp1A1 gene expression and cytotoxic response to PAHs, an intriguing and attractive concept that is undergoing further investigation.

Acknowledgments: This study was supported, in part, by research funds from NIH/RCMI Grant \# G12RR03062. This article is based on a presentation at the $2^{\text {nd }}$ International Symposium on Recent Advances in Environmental Health Research, commemorating the $20^{\text {th }}$ anniversary of the National Institutes of Health Research Centers in Minority Institutions (RCMI) Program, hosted by Jackson State University, Jackson, Mississippi, September 18-21, 2005.

\section{References}

1. Okey, A B. Enzyme induction in the cytochrome P-450 system. Pharmacol Ther., 1990, 45, 241-298.

2. Guengerich, F.P. Molecular advances for the cytochrome P-450 superfamily. Trends Pharmacol Sci., 1991, 12:281-283.

3. Perdew, G. H. Association of the Ah receptor with the 90-kDa heat shock protein. J Biol Chem., 1988, 263, 13802-13805.: 
4. Chen, H. S.; Perdew, G. H. Subunit composition of the heteromeric cytosolic aryl hydrocarbon receptor complex. J Biol Chem., 1994, 269, 27554-27558.

5. Whitlock, J.; Okino, S. T.; Dong, L.; Ko, H. P.; Clarke-Katzenberg, R.; Ma, Q.; Li, H.: Induction of cytochrome P450 1A1: a model for analyzing mammalian gene transcription. FASEB J., 1996, 10, 809-818

6. Whitlock, J.: Induction of cytochrome P450 1A1. Annu. Rev. Pharmacol. Toxicol., 1999, 39,103-125.

7. Pollenz, R.S.; Sattler, C.A. and Poland, A. The aryl hydrocarbon receptor and aryl hydrocarbon receptor nuclear translocator protein show distinct subcellular localizations in Hepa 1c1c7 cells by immunofluorescence microscopy. Mol. Pharmacol., 1994, 45, 428-438.

8. Hankinson, O. The aryl hydrocarbon receptor complex. Annu Rev Pharmacol Toxicol., 1995, 35, 307-340.

9. Merrill Jr., A. H.: Characterization of serine palmitoyltransferase activity in Chinese hamster ovary cells. Biochim Biophys Acta., 1983, 754, 284-291.

10. Merrill Jr., A. H.; Schmelz, E.-M.; Dillehay, D. L.; Spiegel, S.; Shayman, J. A.; Schroeder, J. J.; Riley, R. T.; Voss, K. A.; Wang, E.: Sphingolipids-The enigmatic Lipid Class: Biochemistry, Physiology, and Pathophysiology. Toxicol. and Applied Pharmacol., 1997, 142, 208-225.

11. Williams, R. D.; Wang, E.; Merrill, A H.: Enzymology of long-chain base synthesis by liver: characterization of serine palmitoyltransferase in rat liver microsomes. Arch. Biochem. Biophys., 1984, 228, 282-291.

12. Merrill Jr., A. H.: De novo sphingolipid biosynthesis: a necessary but dangerous pathway. J. Biol. Chem., 2002, 277, 25843-25846.

13. Nikolova-Karakashian, M. N.; Morgan, E. T.; Alexander, C.; Liotta, D. C.; Merrill Jr., A. H.: Bimodal regulation of ceramidase by interleukin-1B: Implication for the regulation of cytochrome $\mathrm{P} 450 \quad 2 \mathrm{C} 11$ (CYP2C11) J. Biol. Chem., 1977, 272, 18718-18724.

14. Merrill Jr., A. H., Morgan, E.T., NikolovaKarakashian, M. and Stewart, J. Sphingomyelin hydrolysis and regulation of the expression of the gene for cytochrome P450. Biochem Soc Trans., 1999, 27, 383-387.

15. Huwiler, A.; Kolter, T.; Pfeilschifter, J.; Sandhoff, K.: Physiology and pathophysiology of sphingolipid metabolism and signaling. Biochimica et Biophysica Acta., 2000, 1485, 63-99.

16. Wang, H.; Maurer, B. J.; Reynolds, C. P.; Cabot, M. C.: N-(4-hydroxyphenyl)retinamide elevates ceramide in neuroblastoma cell lines by coordinate activation of serine palmitoyltransferase and ceramide synthase. Cancer Res., 2001, 61, 5102-5105.

17. Dedov, V. N.; Dedova, N.; Nicholson, G. A.: Hypoxia causes aggregation of serine palmitoyltransferase followed by non-apoptotic death of human lymphocytes. Cell Cycle, 2004, 3, 1271-1277.
18. Fradette, C.; du Souich, P.: Effect of hypoxia on cytochrome P450 activity and expression. Current Drug Metab., 2004, 5:257-271.

19. Chen, Z-H.; Hurh, Y-J.; Na, H-K.; Kim, J-H.; Chun, YJ.; Kim, D-H.; Kand, K-S.; Cho, M-H.; Surh, Y-J. Resveratrol inhibits TCDD-induced expression of CYP1A1 and CYP1B1 and cathecol estrogen-mediated oxidative DNA damage in cultured human mammary epithelial cells. Carcinogenesis, 2004, 25, 2005-2013.

20. Carlson, D. B.; Perdew, G. H.: A dynamic role for the Ah receptor in cell signaling? Insights from a diverse group of Ah receptor interacting proteins. $J$ Biochem Mol Toxicol. 2002; 16(6):317-25.

21. Levine-Fridman, A.; Chen, L.; Elferink, C. J.: Cytochrome P4501A1 promotes G1 phase cell cycle progression by controlling aryl hydrocarbon receptor activity. Mol Pharmacol., 2004, 65, 461-469.

22. Batheja, A. D.; Uhlinger, D. J.; Carton, J. M.; Ho, G.; D'Andrea, M. R: Characterization of serine palmitoyltransferase in normal human tissues. $J$. Histochem. Cytochem., 2003, 51, 687-696.

23. Bowen, W. P.; Carey, J. E.; Miah, A.; McMurray, H. F.; Munday, P. W.; James, R. S.; Coleman, R. A.; Brown, A. M.: Measurement of cytochrome P450 gene induction in human hepatocytes using quantitative realtime reverse transcriptase-polymerase chain reaction. Drug Metab Dispos., 2000, 28, 781-8.

24. Weiss, B.; Stoffell, W.: Human and murine serinepalmitoyltransferase- cloning, expression and characterization of the key enzyme in sphingolipid synthesis. Eur. J. Biochem., 1997, 249, 239-247.

25. Cody, C. W.; Prasher, D. C.; Westler, W. M.; Prendergast, F. G.; Ward, W. W.: Chemical structure of the hexapeptide chromophore of the Aequorea greenfluorescent protein. Biochemistry, 1993, 32, 1212-8.

26. Sullards, M. C.; Wang, E.; Peng, Q.; Merrill Jr., A. H.: Metabolomic profiling of sphingolipids in human glioma cell lines by liquid chromatography tandem mass spectrometry. Cell. Mol. Biol., 2003, 49, 789-797.

27. Kok J. W.; Veldman, R. J.; Klappe, K.; Koning, H.; Filipeanu, C. M.; Muller, M.: Differential expression of sphingolipids in MRP1 overexpressing HT29 cells. Int J Cancer, 2000, 87, 172-178.

28. Yun, C-O.; Park, H-J.; Kim, S-J.; Kim, H-K.: Identification of cytochrome P-450 1A1 in human brain. Biochem. Biophys. Res. Commun., 1998, 243, 808-810.

29. Crofts, F. G.; Sutter, T. R.; Strickland, P. T.: Metabolism of 2-amino-1-methyl-6-phrnylimidazo [4,5-b] - pyridine by human cytochrome P4501A1, $\mathrm{P} 4501 \mathrm{~A} 2$ and P4501B1. Carcinogenesis, 1998, 19, 1969-1973.

30. Pratt, W. B.; Toft, D. O.: Regulation of signaling protein function and trafficking by the Hsp90/Hsp70based chaperone machinery. Exp. Biol. Med., 2003, 228, 111-133.

31. Meyer, B. K.; Petrulis, J. R.; Perdew, G. H.: Aryl hydrocarbon (Ah) receptor levels are selectively 
modulated by hsp90-associated immunophilin homolog XAP2. Cell Stress Chaperones, 2000, 5, 243-254.

32. Kazlauskas, A.; Poellinger, L.; Pongratz, I.: The immunophilin-like protein XAP2 regulates ubiquitination and subcellular localization of the dioxin receptor. J Biol Chem., 2000, 275, 41317-41324.

33. Riboni, L.; Campanella, R.; Bassi, R.; Villani, R.; Gaini, S. M.; Martinelli-Boneschi, F.; Viani, P.;
Tettamanti, G.: Ceramide levels are inversely associated with malignant progression of human glial tumors. Glia, 2002, 39, 105-13.

34. Ogretmen, B.; Hannun, Y. A.: Biologically active sphingolipids in cancer pathogenesis and treatment. Nat Rev Cancer, 2004, 4, 604-616.

35. Holland, E. C.: Glioblastoma multiforme: The terminator. Proceedings Natl. Acad. Science (USA), 2000, 97, 6242-6244. 